\title{
SEM characterization of two-dimensional patterns generated in titanium by femtosecond laser interferometry
}

\author{
V. Oliveira*****, N.I. Polushkin ${ }^{* * * * * * * * * *}$, O. Conde ${ }^{* * *, * * * *}$ and R. Vilar**,**** \\ *Instituto Superior de Engenharia de Lisboa, Avenida Conselheiro Emídio Navarro no 1, 1959-007 Lisboa, \\ Portugal \\ **Instituto Superior Técnico, Avenida Rovisco Pais no 1, 1049-001 Lisboa, Portugal \\ ***Faculdade de Ciências da Universidade de Lisboa, Campo Grande Ed. C8, 1749-016 Lisbon, Portugal \\ ****Instituto de Ciência e Engenharia de Materiais e Superfícies, Avenida Rovisco Pais no 1, 1049-001 Lisboa, \\ Portugal
}

Laser ablation using ultrafast femtosecond lasers and holographic schemes has proven to be a powerful and versatile tool for surface and volume structuring. The principle of operation of this technique is simple: when two or more pulses overlap in time and space, an interference pattern is generated that can be used to create periodic surface structures. In addition, due to the extremely short pulse duration, a very high peak power is achieved leading to intense non-linear effects. As a result, almost any type of material can be processed without undesirable collateral thermal effects.

In this paper, characterization of two-dimensional (2D) patterns generated in titanium using femtosecond laser radiation has been carried out using scanning electron microscopy (SEM). The laser source is a commercial $\mathrm{Yb}: \mathrm{KYW}$ laser system providing pulses with a duration of $560 \mathrm{fs}$ at a central wavelength of $\lambda=1030 \mathrm{~nm}$. The surface topography was characterized using a Hitachi S2400 scanning electron microscope operated at an electron acceleration voltage of $25.0 \mathrm{kV}$. Laser processing was performed in air on polished grade 2 titanium samples, a material typically used in low load bearing medical devices.

One-dimensional (1D) gratings were created using a modified Michelson interferometer described in detail elsewhere [1]. To create 2D gratings a double exposure method was used. First, 1D gratings were produced in linear tracks by translating the sample relatively to the stationary interfering laser beams with a fixed scanning velocity of $0.1 \mathrm{~mm} / \mathrm{s}$. As an example, Figure 1 depicts SEM pictures of horizontal and vertical $1 \mathrm{D}$ gratings with period of about $3.9 \mu \mathrm{m}$, generated using a pulse energy and pulse repetition rate of $0.35 \mathrm{~mJ}$ and $100 \mathrm{~Hz}$, respectively. The peak to valley distance of these patterns can be controlled either by changing the scanning velocity or the pulse repetition rate. By overlapping two linear tracks, different kinds of 2D structures can be created. Figure 2 depicts a square pattern obtained by overlapping two $1 \mathrm{D}$ gratings rotated by $90^{\circ}$. The dimensions of the squares depend on the one-dimensional gratings period, which in turn can be easily controlled by varying the distance between the interfering beams. Figure 3 depicts two other possibilities: i) trapezium-like patterns obtained by rotating the $1 \mathrm{D}$ gratings by $45^{\circ}$, and ii) rectangular patterns obtained using $1 \mathrm{D}$ gratings with different periods and rotated by $90^{\circ}$.

The proposed optical setup offers a simple method of texturing the surface of materials and, hence, to control surface properties such as wettability. In the case of titanium, this is particularly important because surface texturing enhances its osseointegration ability. For this purpose, when compared with the columns spontaneously formed on titanium surfaces treated with femtosecond laser radiation [2], these 2D gratings present the major advantage of being size and shape-controllable.

\section{References}

1. Oliveira V. et al., Opt. Las. Techn., 44:2072, 2012.

2. Oliveira V. et al., Appl. Surf. Sci., 255:7560, 2009. 

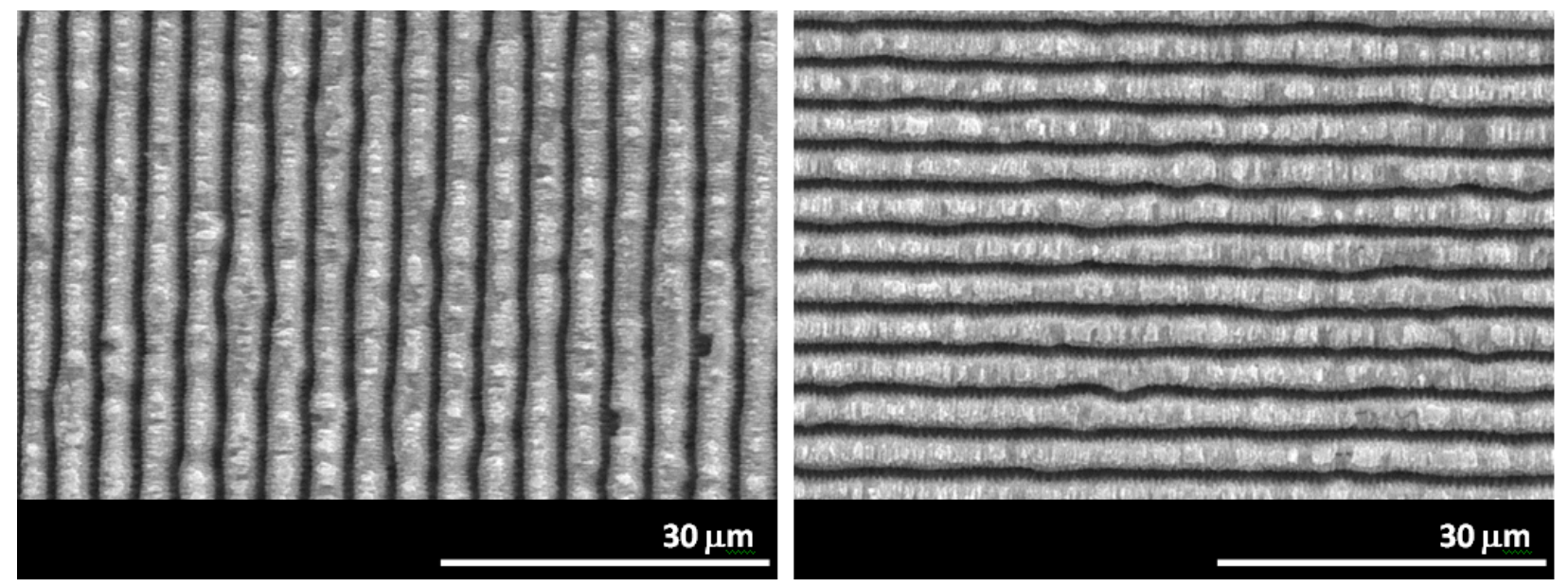

Figure 1. SEM images of 1D patterns generated by single exposure.
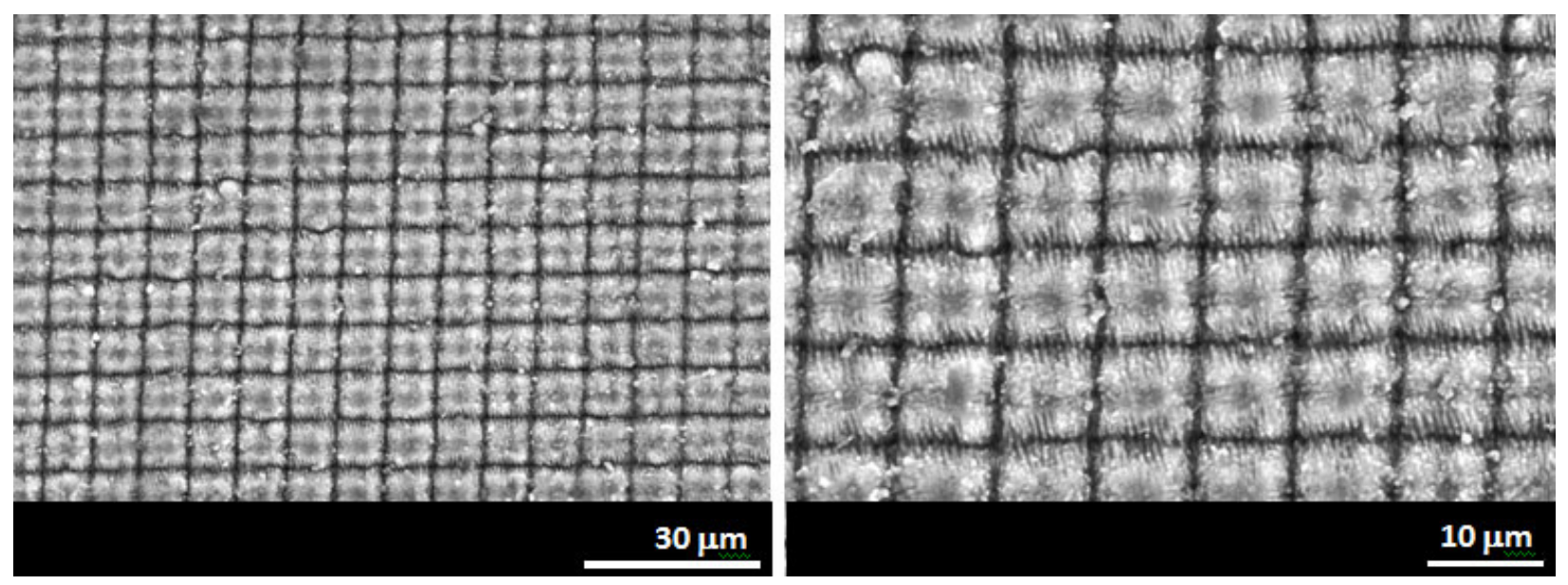

Figure 2. SEM images of a 2D square pattern generated by overlapping two $1 \mathrm{D}$ gratings rotated by $90^{\circ}$.
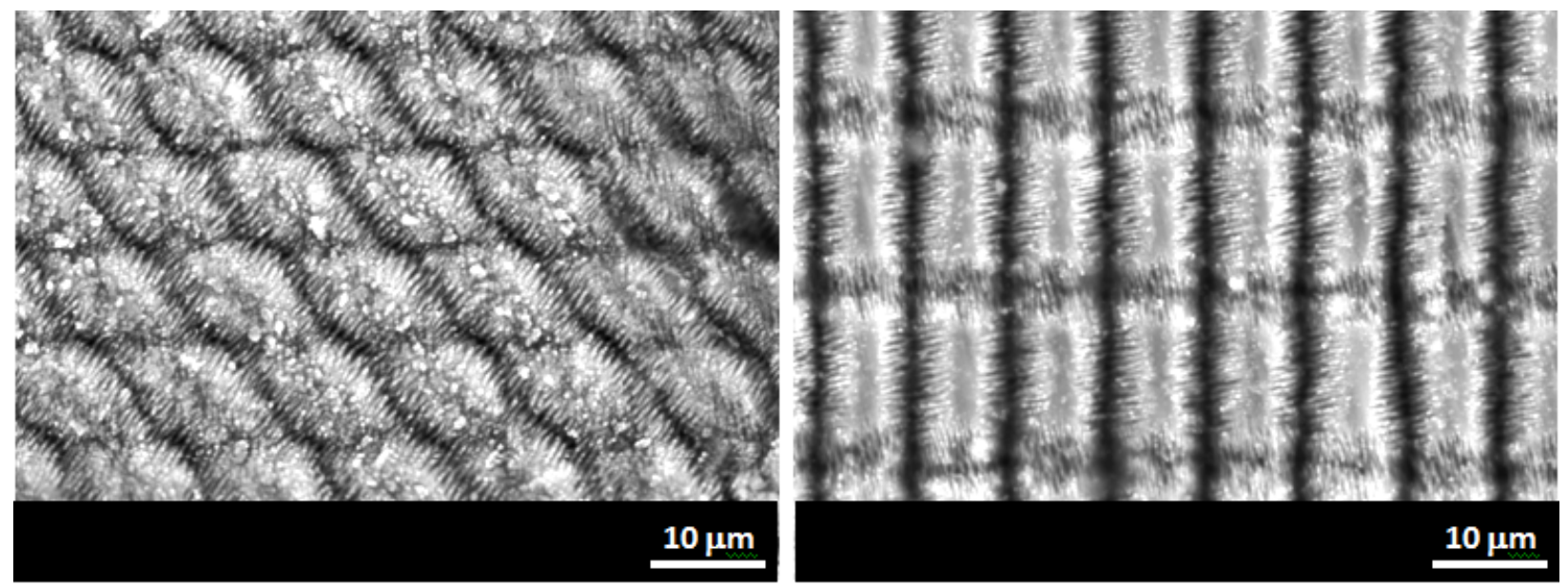

Figure 3. SEM image of 2D patterns generated by double exposure. 